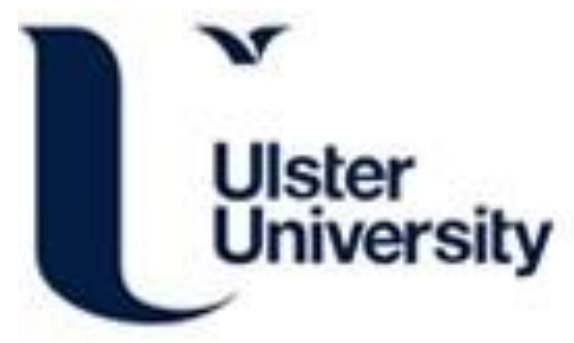

\title{
Prosthesis grasp reflex via peripheral nerve control - an in vitro study
}

Valderrama, G., Gasson, M. N., Warwick, K., \& Whalley, B. J. (2010). Prosthesis grasp reflex via peripheral nerve control - an in vitro study. In Unknown Host Publication (pp. 1-6). IEEE. https://doi.org/10.1109/UKRICIS.2010.5898090

Link to publication record in Ulster University Research Portal

\section{Published in:}

Unknown Host Publication

Publication Status:

Published (in print/issue): 02/09/2010

DOI:

10.1109/UKRICIS.2010.5898090

\section{Document Version}

Publisher's PDF, also known as Version of record

\section{General rights}

Copyright for the publications made accessible via Ulster University's Research Portal is retained by the author(s) and / or other copyright owners and it is a condition of accessing these publications that users recognise and abide by the legal requirements associated with these rights.

\section{Take down policy}

The Research Portal is Ulster University's institutional repository that provides access to Ulster's research outputs. Every effort has been made to ensure that content in the Research Portal does not infringe any person's rights, or applicable UK laws. If you discover content in the Research Portal that you believe breaches copyright or violates any law, please contact pure-support@ulster.ac.uk. 


\section{Prosthesis grasp reflex via peripheral nerve control - an in vitro study}

\author{
German D. Valderrama-Gonzalez \\ School of Computing and Intelligent Systems \\ University of Ulster \\ Derry, UK \\ g.valderrama@ulster.ac.uk
}

\author{
Mark N. Gasson \\ and Kevin Warwick \\ School of Systems Engineering \\ University of Reading \\ m.n.gasson@ reading.ac.uk \\ k.warwick@reading.ac.uk
}

\author{
Benjamin J. Whalley \\ School of Pharmacy \\ University of Reading \\ Reading, UK \\ b.j.whalley@reading.ac.uk
}

\begin{abstract}
Here we present an economical and versatile platform for developing motor control and sensory feedback of a prosthetic hand via in vitro mammalian peripheral nerve activity. In this study, closed-loop control of the grasp function of the prosthetic hand was achieved by stimulation of a peripheral nerve preparation in response to slip sensor data from a robotic hand, forming a rudimentary reflex action. The single degree of freedom grasp was triggered by single unit activity from motor and sensory fibers, identified and classified according to their speed of conduction, as a result of stimulation. The work presented here provides a novel, reproducible, economic, and robust platform for experimenting with neural control of prosthetic hand grasp before translating into in vivo implementation.
\end{abstract}

\section{INTRODUCTION}

Researchers have long sought ways to create interfaces to the human body which can restore motor and sensory function in patients with severe paralysis caused by disease or injury [1], [2]. It was estimated by the National Institute of Health (NIH) that in the U.S. alone there are 600,000 new cases of stroke a year [3], 250,000 people living with spinal cord injury, with 10,000 to 12,000 new cases a year [4], [5], 1.7 million people with limb loss [6], [7] with 130,000 non-traumatic lower limb amputations each year [8], [9]. When considering potential therapies, establishing communication between the nervous system and a device such as prosthesis is of key importance.

From a biological perspective, peripheral nerve signals can be divided in two groups: afferent sensory signals that provide feedback and motor signals that perform control (e.g. limb movement). Consequently, two interface types between biological and machine systems may be considered; the first includes sensory prostheses such as the cochlear implant, the vestibular implant, implanted vision prostheses, brain stimulation for pain control, cranial nerves stimulation for epileptic seizure control and deep brain stimulation for control Parkinsonian symptoms. The second type is the neuromotor prostheses.

"Neuromotor prostheses are a type of brain-machine interface that extract signals from the central nervous system (CNS) or peripheral nervous system (PNS) and deliver them to control devices" [10].

Whether investigating peripheral or central nervous system bioelectrical activity, a common feature is the detection and recording of axonal conduction of action potentials (APs) an electrode or array of electrodes placed proximal to activity source before being transformed and stored as a digital signal. Usually, the command signals for upper limb-implanted prostheses are obtained via electromyogram (EMG) [11], intracortical electrode arrays [12], [13], surface cortical potentials (e.g. electroencephalography (EEG) and electrocorticography (ECoG); see [14], [15], [16] for reviews). However, this [17], [18] and other two research groups [19], [20] have successfully taken the signal from peripheral nerve electrophysiological recordings. A neuroprosthesis with command signals derived from peripheral nerve activity employing such an interface can provide rapid feedback [17], [18], [19], [20] and may offer a more intuitively natural control mechanism to facilitate prosthesis integration into the user's body image [19]. Moreover, a more natural control of the first DOF by a single electrode in a nerve reflex type manner as present here could enhance in vivo implantation of upper-limb prostheses.

Here, we describe control of a single-degree of freedom (DOF) prosthetic hand [21], [22], [23] via evoked and electrophysiologically recorded APs acquired from a rat sciatic nerve preparation, in vitro [24]. Briefly, activation of the prosthesis fingertip sensors triggers electrode-mediated nerve stimulation resulting in neural activity that travels in both orthodromic and antidromic directions to be recorded via a second electrode at the distal nerve end. The neural signal is then amplified and filtered before being used as a control input to the prosthesis to activate the closing reflex. Thus, in an in vivo implementation, pressure sensor activation could provide sensory feedback to the user and augment motor control by the user to provide reflexive opening and closing and so improving interaction quality and reaction time.

However, in vivo testing of prototyping prosthesis have several problems such as ethical approval, biocompability, biostability, useful time span of the preparation and difficulty on completing the loop because the sensory pulse coming from the prosthetic hand would have to travel up to the brain and downwards to the animal limb with the motor signal order to close the hand which increase the reaction time and 


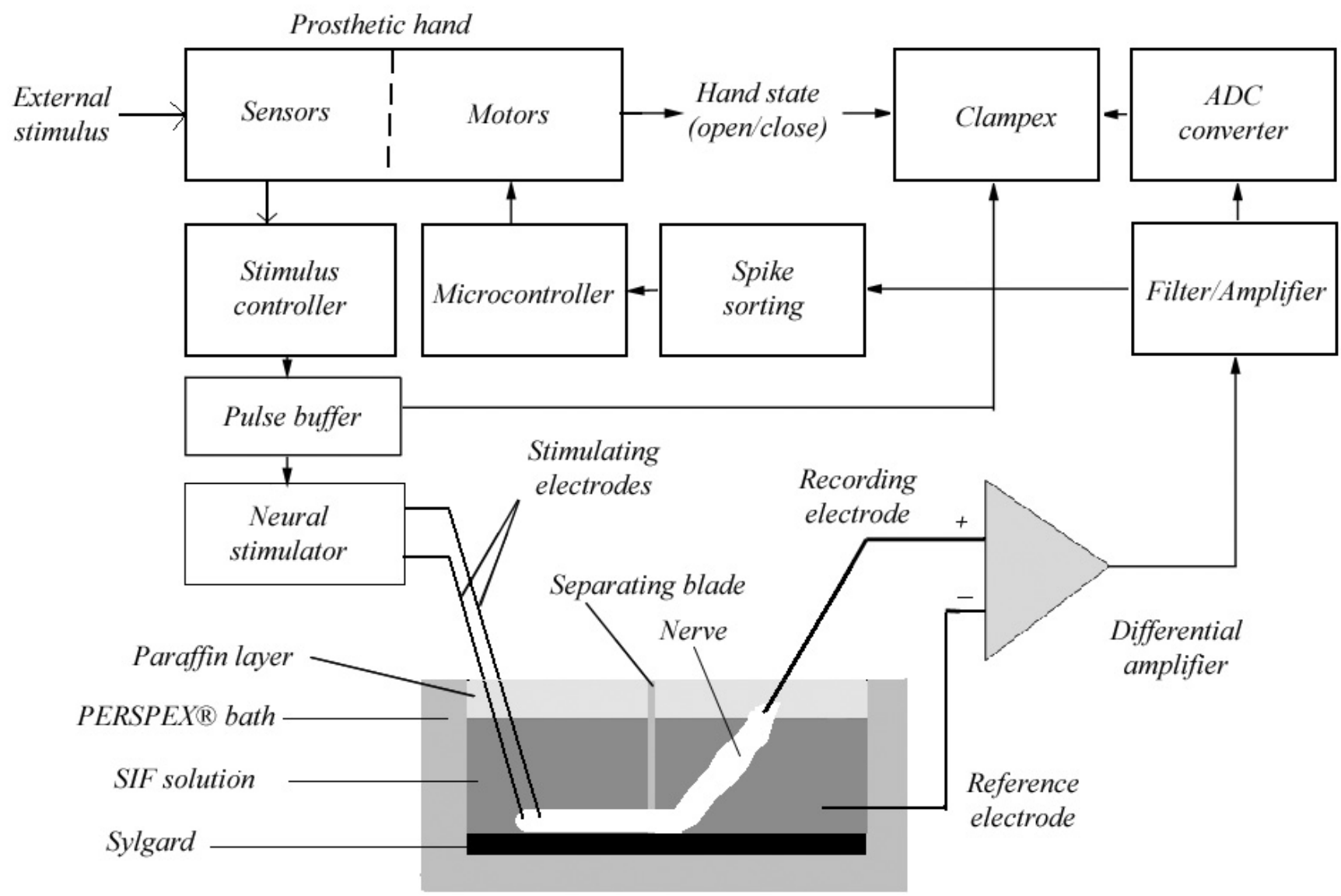

Fig. 1. Diagrammatic illustration of the system employed to provide a feedback loop to control a robotic hand via an in vitro peripheral nerve.

add noise. On the other hand, the novel work presented here provides a stable, economic, rapid, and robust platform for prototyping such devices offering a neural mechanism to control the opening and closing of the hand at a peripheral level without requiring processing of the information by the brain. Thus, the brain is free to control other aspects of the prosthesis. Achieving this in a reproducible manner is fundamentally important before attempting it in vivo.

\section{IMPLEMENTATION AND METHODS}

A. In vitro nerve preparation, bath and physiologically relevant maintenance solution

Outbred 250 - 300g male and female Lister Hooded rats were euthanized by overdose of inhalant anaesthetic (Isoflurane-vet, Merial, UK) and sacrificed by a Schedule 1 method. All experiments conformed to the UK Animals (Scientific Procedures) Act 1986 and every effort was made to minimise the number of animals used. Animals were shaved prior to dissection to prevent conducting hair fiber contamination. The sciatic nerve dissection has been described elsewhere in detail [25]. Briefly, small bilateral inguinal incisions were then made and the skin separated from the body. The dorsum and limbs were separated from the rest of the body and placed in a Petri dish containing a synthetic interstitial fluid (SIF) of composition: (in $\mathrm{mM}$ ): $\mathrm{NaCl} 139.7, \mathrm{KCl} 3.5, C a C L_{2} 1.26$, dglucose 10, $\mathrm{NaHCO}_{3} 20.9, \mathrm{NaH}_{2} \mathrm{PO}_{4} 0.65, \mathrm{MgCl}_{2} 6.0$ and $\mathrm{H}_{2} \mathrm{O}$ 1.0. The SIF was constantly carboxygenated $\left(95 \% \mathrm{O}_{2} /\right.$ $\left.5 \% \mathrm{CO}_{2} ; \sim 3 \mathrm{~L} / \mathrm{min}\right)$. All solutions were freshly prepared each day and $\mathrm{pH}(7.2$ - 7.6) monitored before and during (every 2 hours) experimentation and adjusted if necessary by replacing the solution.

The femoral and sciatic artery and vein were then identified and dissected away from the lumbosacral trunk that contains the sciatic and pudendal nerves. Then, beginning at the lumbar roots, the lumbosacral trunk was cleared of surrounding fascia and the pudendal nerve gently separated from the sciatic nerve which was subsequently ligated (Johnson \& Johnson, US) at both ends before being gently separated from surrounding structures. Physical manipulation of the nerve was conducted with care to preserve axonal integrity. The dissection was repeated for the contralateral side and thus typically yielded two sections of sciatic nerve of $\sim 4.5 \mathrm{~cm}$ length. The isolated nerves were then placed in a SIF-filled Petri dish that had been lined with black sylgard and inspected using a 50x binocular microscope (Wild Heerbrugg, Switzerland). At one end, the 


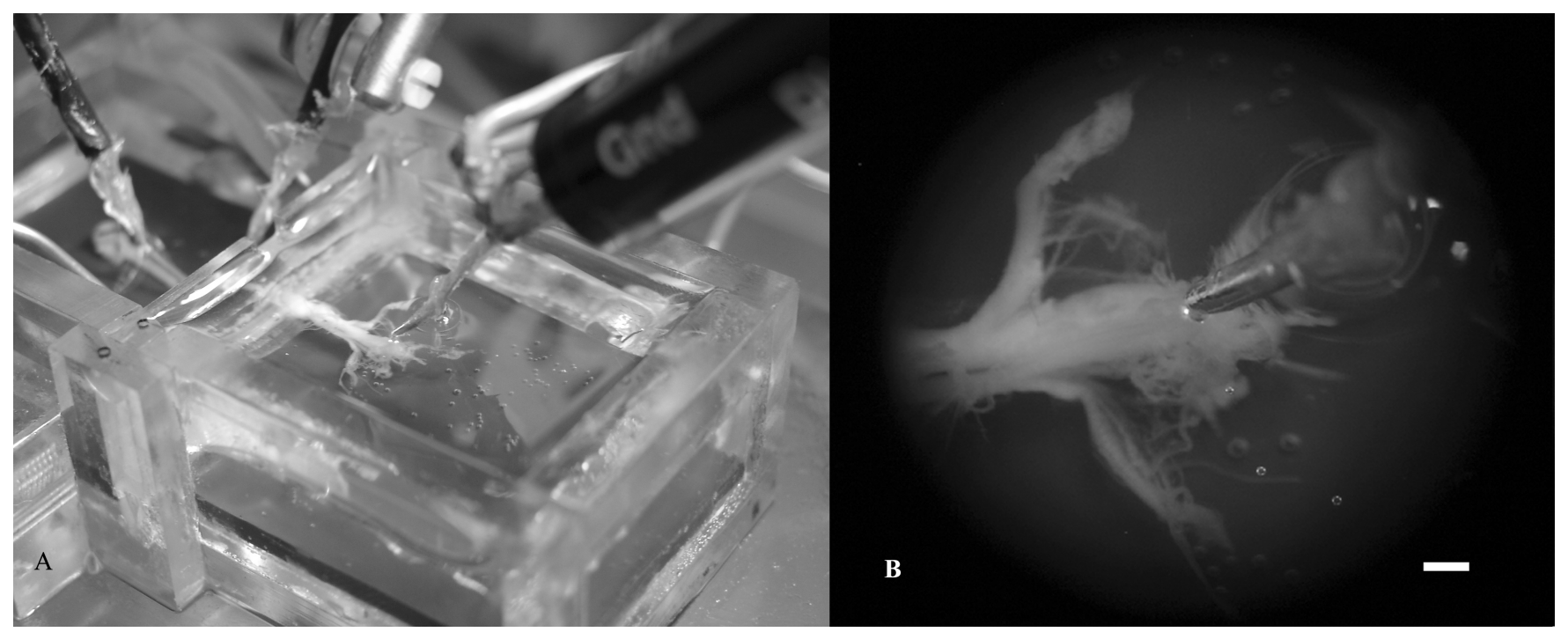

Fig. 2. Electrophysiological set up. (A) Two chamber grease gap bath with nerve preparation. Two stimulating electrodes are positioned on the left chamber. Two recording electrodes on the right, one connected to the headstage and a reference electrode just immersed on the SIF solution at the right corner. (B) Magnification of de-sheathed distal nerve end. Bar line $\sim 200 \mu \mathrm{m}$.

ligature and $\sim 1.5 \mathrm{~cm}$ of the myelin sheath was removed from one end of each nerve using superfine straight and angled Vannas scissors, forceps 5.s (Ideal-tek, Switzerland) and a fine scalpel (Fine Science Tools, Inc., Canada) to expose the fascicules and this end was ligated again. The tissue was then immersed in a SIF-containing bath for $\geq 1$ hour to recover before use. Under these conditions, an isolated sciatic nerve could be maintained for $7 \pm 3 \mathrm{hrs}(\mathrm{n}=200)$.

In order to provide a suitable maintenance and recording environment, a two chamber grease-gap bath [26] was constructed to allow gravity fed SIF exchange (maintained at $30^{\circ} \mathrm{C} \pm 1^{\circ} \mathrm{C}$ ) and electrical isolation of the recording and stimulation chambers from one another (Figure 1). Thus, current flow from one chamber to the other could only occur via the isolated sciatic nerve whilst minimising stimulationinduced artifacts. Bath chambers were separated by a $1 \mathrm{~mm}$ partition blade with a small notch to allow unimpeded passage of nerve tissue between chambers (Figure 2). Silicone grease (Edwards High Vacuum Ltd, UK) was used to ensure electrical and fluid isolation between compartments at the point at which the nerve passed through the partition blade. The SIF within the recording bath was topped with a thin layer of high resistance paraffin (Merck, UK) in which the distal nerve end was suspended to provide the recording amplifier with a differential between the two recording electrodes (see also section B). The recording system was situated inside a Faraday cage and mounted on a MICRO-g anti-vibration table (Technical Manufacturing Corporation, US) to prevent interference from the ambient recording environment.

\section{B. Electrophysiological recording}

A pre-amplifier headstage (NL100AK; Digitimer, UK) was used to provide the differential between a SIF-immersed reference electrode and the stainless steel recording electrode that was placed proximal to the exposed nerve fibers in the paraffin layer of the bath. The recording electrode shaft was insulated with silicone except at the $\sim 50-80 \mu \mathrm{m}$ diameter tip. Fine positioning of the recording electrode was achieved using a micromanipulator (Prior, UK) under binocular microscope observation.

The amplitude of extracellularly recorded APs are typically of the order of microvolts, necessitating headstage connection to an amplifier with 1000x - 5000x gain (NL104A; Digitimer, UK) and bandpass $(10 \mathrm{~Hz}-10 \mathrm{KHz})$ and notch $(50 \mathrm{~Hz})$ filters (NL125; Digitimer, UK). Output was split to provide input to an analogue-to-digital data capture card (Digidata 1200) and a 54600B digital oscilloscope (Hewlett Packard, U.S.) for visualisation and a gated amplitude discriminator ('spike trigger'; NL 201; Digitimer, UK). The spike trigger threshold level was set at $3 x$ the SNR. The spike trigger produces a pulse whenever APs were present, detecting even single spike events. Clampex (Molecular Devices, US), LTP Program (version 2.4; WinLTP Ltd, UK) and Picoscope (Pico Technology, UK) capture device were used to save recordings at sampling rates of $333 \mathrm{KHz}$, $10 \mathrm{KHz}$ and $1 \mathrm{KHz}$ respectively.

Typical axonal diameter is of the order of $<20 \mu \mathrm{m}$. Given the $50-80 \mu \mathrm{m}$ diameter of the recording electrode and the gross stimulation method employed, the recordings made were likely to originate from fiber sub-populations and so represent compound action potentials [27]. While it is possible to extract single units from extracellular recordings via spike sorting in either the time or frequency domains [28], this is not easily achieved with recordings containing high levels of correlated signal and superposition of waveforms such as is the present case. Since the sciatic nerve is connected to the ventral (motor) and dorsal (sensory) roots of L4, L5, S1, S2, and S3, the nerve contains sural, peroneal and tibial fascicules. Each fascicule contains hundreds of different axon types that 
exhibit different conduction speeds. Consequently, temporal clustering of activity was used to discriminate between fiber systems.

\section{The prosthetic hand}

The type of articulated hand prosthesis employed in this study has multiple degrees of freedom and can be controlled in a hierarchical manner. The SNAVE hand [21], [22], [23] (also called the Southampton Hand; Figure 3) uses fingertip slip sensors to react to environmental stimuli and joint angle sensors for control. Joint flexion and force sensors allow adaptation of grip shape and for the force applied to a grasped object to be modified. Using the on-board microcontroller, the hand can automatically ensure that the lightest possible touch is applied and the object is grasped in the most appropriate manner. This has previously been achieved via EMG signals, making it a suitable platform for testing the present work.

The stable state of the hand's position was open. The hand was programmed such that when the finger-tip pressure sensors of the SNAVE hand touched an object, the hand sent a pulse to a pulse buffer unit (NL510; Digitimer, UK), a stimulus controller that set stimulation parameters and trigger a neural stimulator (NL800 stimulus isolator; Digitimer, UK). Stimulus amplitudes sufficient to produce a significant response were used but did not exceed $100 \mu \mathrm{A}$. Constant current stimulation (5-15 ms pulse) at the proximal nerve end was delivered via two silicone insulated stainless steel electrodes positioned on either side of the nerve trunk. In response to the stimulation some fibers of the nerve fires and resulting nerve activity was recorded by the previously described headstage.

Amplified and filtered neural signal was used as input to the prosthesis via the spike trigger. In the presence of APs, the spike trigger sent a pulse to the hand's microcontroller. Each and every spike trigger's pulse increased the microcontroller's voltage. When certain voltage threshold was reached, the grasp would complete using the on-board intelligence of the hand to adopt the most appropriate grip, which it would hold for 2 seconds and then release, unless subsequent activation occurred, in which case the grasp would be held. As such, an object touching the finger-tips causes the hand to close around it, and the grasp would be maintained until the object was removed from the grasp.

\section{RESULTS}

\section{A. Validation of the electrophysiological recordings}

In order to validate the system, a periodic stimulus (50 $100 \mu \mathrm{A} ; 5-15 \mathrm{~ms} ; 0.2 \mathrm{~Hz}$ ) was applied until activity was evident or the preparation was assessed as not viable and discarded. Figure $4 \mathrm{a}$ shows the response of an isolated nerve to a delivered stimulus $(60 \mu \mathrm{A} ; 5 \mathrm{~ms} ; 0.2 \mathrm{~Hz})$. Temporally clustered neural activity can be seen occurring $6,45,53$ and $78 \mathrm{~ms}$ after the onset of stimulation. In order to validate that the recordings were of a physiological nature and not stimulation artifacts, tetrodotoxin (TTX; 1M; a neurotoxin that blocks sodium channels and therefore prevents the occurrence of APs) was added to the SIF solution. A graded reduction

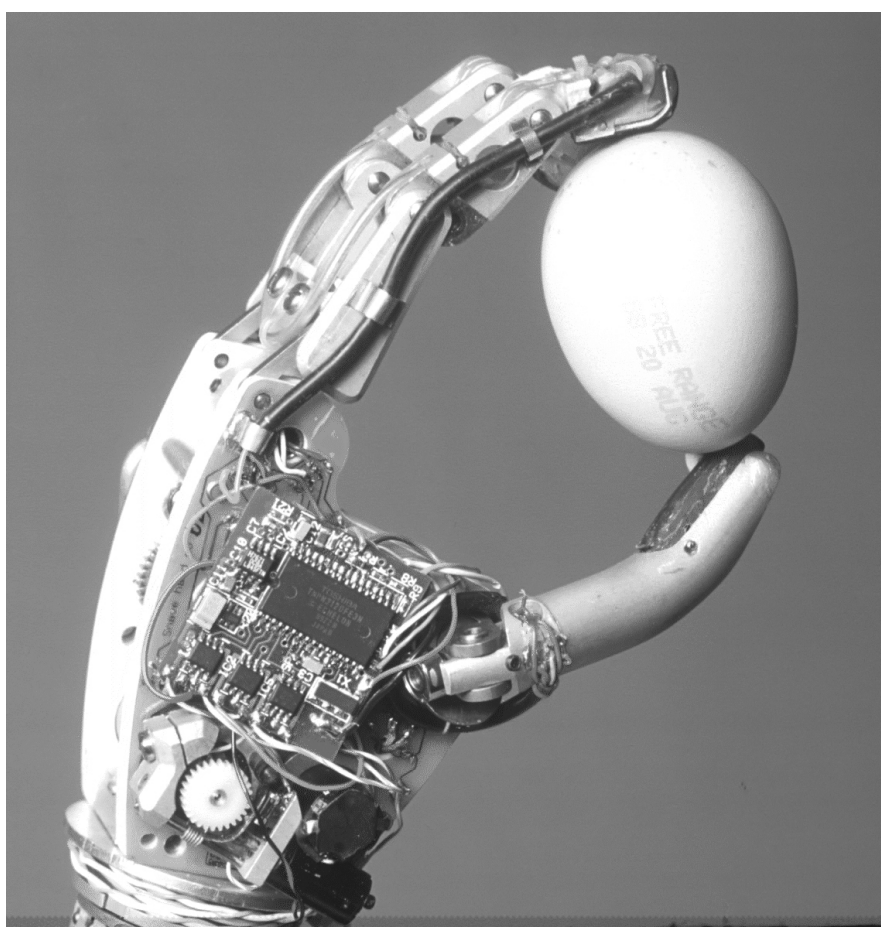

Fig. 3. The SNAVE prosthetic hand using slip sensors in its fingertips to hold an egg with an appropriate force.

in compound spikes was observed before eventual abolition. Figure $4 \mathrm{~b}$ shows recordings from the same nerve obtained ten minutes after the addition of TTX. The observed AP abolition validates these physiological nature of the responses obtained. This also confirms that activity was not caused by simple conduction though the nerve tissue. This is a reference recording that allows the stimulus artifact and noise to be assessed. The setup described facilitated very low noise recordings with a good signal-to-noise ratio (SNR) even with low amplitude signals. The noise level of the system was found to be $5 \mu \mathrm{V}$ peak-to-peak.

\section{B. Electrophysiological grasp reflex of the prosthetic hand}

Using the setup described previously, a ball was presented to the hand. Figure 5 shows a trace representing the grasp position of the hand - between $0 \%$ and $100 \%$. It can be seen that the hand closes to grasp the ball at around $1 \mathrm{sec}$, the grasp is then held for 2 seconds, before attempting to open again. Detection of the object moving in the hand reinitiates the cycle, maintaining the grasp. The hand was able to maintain grasp for as long as the pressure sensors detected the ball.

\section{DISCUSSION}

Research in rehabilitation prosthetics had focused on myolectric prosthesis control with a recent advent on direct cortical and few studies utilizing neural prosthesis control. This study reaffirms that a third control input, the peripheral nerve control, is possible assuming functionality remains in the severed nerve. It makes sense, as all the information that the brain sends/receives to/from the effector organ -the muscle- 


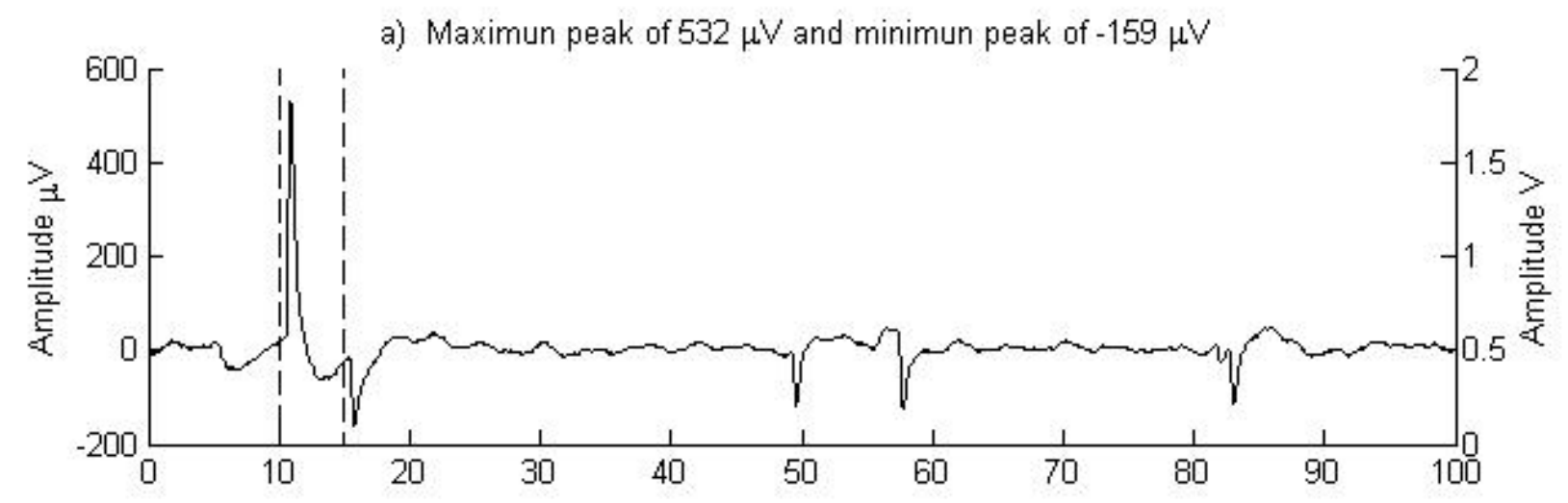

b) Maximun peak of $9 \mu \mathrm{V}$ and minimun peak of $-2 \mu \mathrm{V}$

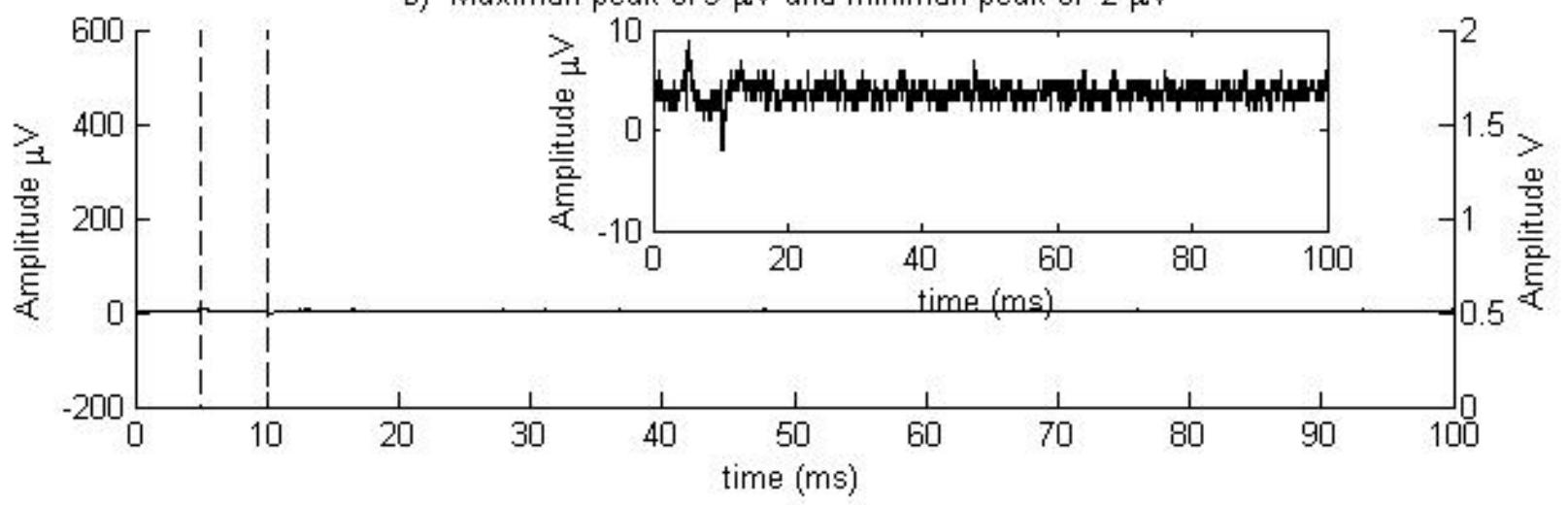

Fig. 4. The top panel shows a representative example of the response of an isolated sciatic nerve following stimulation ( $60 \mu \mathrm{A} ; 5 \mathrm{~ms}$; $10 \mathrm{~ms}$ delay) is shown. Three groups of compound action potentials were observed which occurred 40, 48 and 73 ms after stimulation The bottom panel shows the lack of response to stimulation ( $60 \mu \mathrm{A} ; 5 \mathrm{~ms} ; 10 \mathrm{~ms}$ delay) obtained from the same nerve ten minutes after the application of tetrodotoxin (TTX; $1 \mathrm{M})$. Inset shows that the noise level of the system was found to be $5 \mu \mathrm{V}$ peak-to-peak; the $9 \mu \mathrm{V}$ peak at 5 ms was the stimulus artifact.

travels via peripheral nerve. Results from this study show that it is possible to interface neural tissue to a prosthetic hand providing efferent sensory feedback and afferent motor signals to control it. The sensory feedback was achieved using the prosthetic hand pressure sensors which conducted a current stimulus to the peripheral nerve. As a result of the stimulation both sensory and motor fibers in the nerve fired APs.

The electrophysiological recording setup presented here was remarkable good at reducing noise. Herein, noise isolation techniques permitted to obtain recordings with less than 5 $\mu \mathrm{V}$ peak-to-peak-voltage of noise. An advantage of this was that the motor feedback in this research set up was achieved by attained electrophysiological recordings of the evoked AP sorted by thresholding. Control of the first degree of freedom of the prosthetic hand was accomplished by a rudimentary reflex action: prosthetic hand's stable state was open, upperthreshold AP made the hand close. Multielectrode arrays and a better classification system allowing different grades of opening and closing must be employed in order to achieve a prosthetic hand-human interface. Practical and robust methods of recording and stimulating also need to be developed. However, we present here a novel, simple, robust, rapid, and economic control of a robotic hand in closed loop that included a viable in vitro peripheral nerve preparation. This control did not require higher central nervous system level control, but, instead, required a single recording electrode that utilized peripheral nerve local reflex. Classification of the type of firing fiber based on stimulus strength and firing times is the subject of further work.

\section{CONCLUSIONS}

The work presented in this paper has the following achievements:

- Provides an electrophysiological technique for very low noise recording. This is needed by researchers which preparation requires good signal-to-noise-ratio.

- Use of in vitro peripheral nerve preparation for control of the first degree of freedom of a prosthetic hand in a close loop and in real-time. Achieving this in a reproducible manner is fundamentally important before attempting it in vivo.

- Opening and close of the prosthetic hand was achieved by a rudimentary reflex action that did not require the brain processing. Thus, potentially leaving the brain free to control other aspects of the prosthesis. 


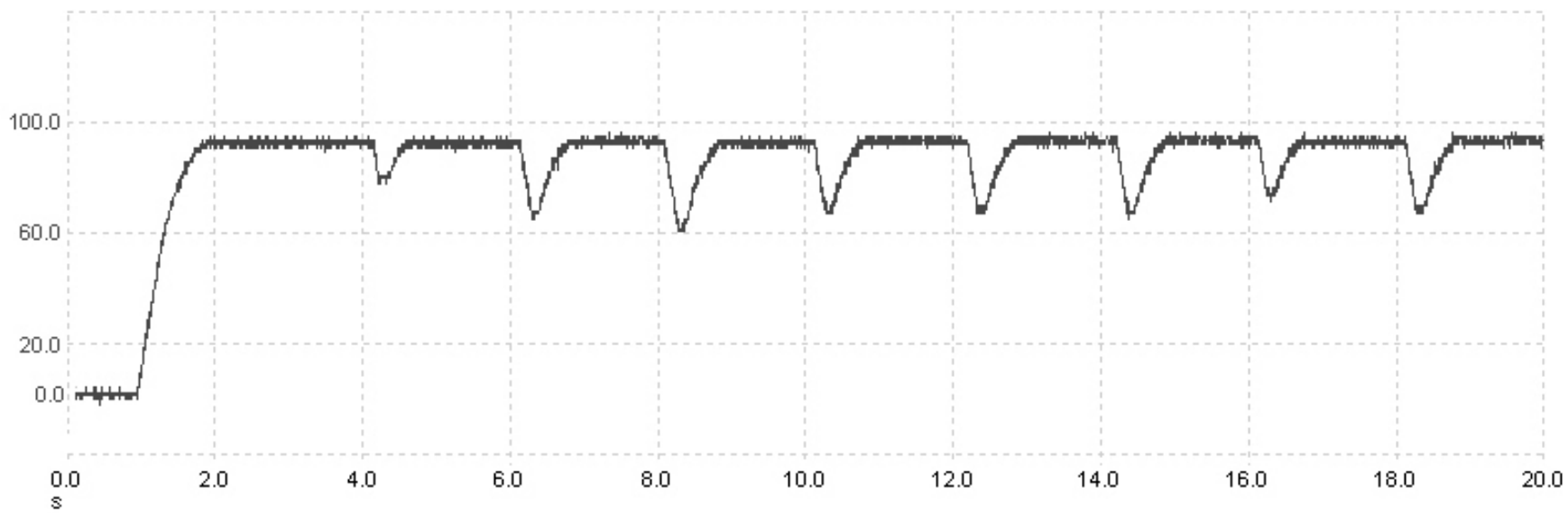

Fig. 5. Representative trace of hand's grasp position over a 20 second period. The hand detects and grasps a ball at $1 \mathrm{~s}$. The hand takes $\sim 1 \mathrm{~s}$ to adopt an appropriate grasp around the object. Every $2 \mathrm{~s}$ thereafter the hand attempts to open but the grasp is maintained through sensor detection of the ball causing nerve stimulation.

\section{ACKNOWLEDGMENT}

We gratefully acknowledge the assistance of Dr. Gayle Passmore and Dr. Steve Marsh, Department of Pharmacology, UCL.

\section{REFERENCES}

[1] J. R. Wolpaw, N. Birbaumer, D. J. McFarland, G. Pfurtscheller and T. M. Vaughan, "Brain Computer Interfaces for Communication and control", Clinical Neurophysiology 113: 767-791,2002.

[2] Nicolelis Miguel A., "Actions from thoughts", Macmillan Magazines Ltd, 2001.

[3] Grotta J. C., Moskowitz M.A. "Report of the stroke progress review group april 2002", National institute of neurological disorders and stroke, 2002.

[4] M. Berkowitz, "Spinal Cord Injury: An Analysis of Medical and Social Costs", Demos Medical Publishing, 1998.

[5] N. (NINDS). (2008, July 24, 2008). "Spinal cord injury: Hope through research". 2008(08/01),

[6] K. Ziegler-Graham, E. J. MacKenzie, P. L. Ephraim, T. G. Travison and R. Brookmeyer, "Estimating the prevalence of limb loss in the United States: 2005 to 2050", Arch. Phys. Med. Rehabil., vol. 89, pp. 422-429, Mar, 2008.

[7] NLLIC, "Amputation Statistics by Cause: Limb Loss in the United States", vol. 2008, 2008.

[8] N. (NIDDK), "National Diabetes Statistics, 2007”, vol. 2008, June 2008, 2008.

[9] National Center for Chronic Disease Prevention and Health Promotion. (December 20, 2005). "National diabetes fact sheet", 2005.

[10] Seruya M and J P Donoghue, "Chapter 7.9 Design Principles of a Neuromotor Prosthetic Device", Advances in Clinical Neurophysiology (Supplements to Clinical Neurophysiology), Vol 57, pp 1158-1196, 2004.

[11] Lipschutz R. D., Kuiken T. A., Miller L. A., Dumanian G. A., Stubblefield K. A., "Shoulder Disarticulation Externally Powered Prosthetic Fitting Following Targeted Muscle Reinnervation for Improved Myoelectric Control", J Prosthet Orthot., Vol 18, pp. 28-34, 2006.

[12] L. R. Hochberg, M. D. Serruya, G. M. Friehs, J. A. Mukand, M. Saleh, A. H. Caplan, A. Branner, D. Chen, R. D. Penn and J. P. Donoghue, "Neuronal ensemble control of prosthetic devices by a human with tetraplegia", Nature 442, 164-171, 2006

[13] D. M. Taylor, S. I. H. Tillery and A. B. Schwartz, "Direct Cortical Control of 3D Neuroprosthetic Devices", Science, vol. 296, pp. 1829, 2002

[14] K. Ohnishi, R. F. Weir and T. A. Kuiken, "Neural machine interfaces for controlling multifunctional powered upper-limb prostheses", Expert Rev.Med.Devices, vol. 4, pp. 43-53, 2007

[15] N. Birbaumer and L. G. Cohen, "Brain-computer interfaces: communication and restoration of movement in paralysis", J. Physiol. (Lond. ), vol. 579, pp. 621, 2007.
[16] M. A. Lebedev and M. A. L. Nicolelis, "Brain-machine interfaces: past, present and future", Trends Neurosci., vol. 29, pp. 536-546, 2006.

[17] Gasson Mark N, "Extending Human Interaction via Invasive Neural Implants", The University of Reading, Reading, U.K., Doctoral Thesis, June 2005.

[18] Warwick K, M Gasson, B Hutt, I Goodhew, P Kyberd, B Andrews, $\mathrm{P}$ Teddy and A Shad, "The Application of Implant Technology for Cybernetic Systems", Archives of Neurology, Vol 60, pp 1369-1373, Oct. 2003.

[19] Dhillon S. G., Horch K. W., "Direct Neural Sensory Feedback and Control of a Prosthetic Arm", IEEE transactions on neural systems and rehabilitation engineering, vol. 13, no. 4, 2005.

[20] X. Jia, M. A. Koenig, X. Zhang, J. Zhang, T. Chen and Z. Chen, "Residual Motor Signal in Long-Term Human Severed Peripheral Nerves and Feasibility of Neural Signal-Controlled Artificial Limb", J. Hand Surg., vol. 32, pp. 657-666, 2007.

[21] Kyberd P J and P H Chappell, "The Southampton Hand: An Intelligent myolectric prosthesis", J Rehabilitation Research and Development, Vol 31, No 4, pp 326 -334, Nov 1994.

[22] Kyberd P, M Evans and S Winkel, "An Intelligent Anthropomorphic Hand, with Automatic Grasp”, Robotica, Vol 16, pp 1531-1536, 1998.

[23] Kyberd, P. J., Light C., Chappell P. H., Nighthingale J. M., Whatley D. and Evans M, "The design of anthropomorphic prosthetic hands: a study of the Southampton hand", Robotica, 19 (6), pp. 593-600, 2001.

[24] Valderrama-Gonzalez G. D., "A study into the peripheral nervous system to control a prosthetic hand", Master Thesis, the University of Reading, Reading, U. K., Oct. 2006.

[25] A. Dalal, M. Tata, G. Allegre, F. Gekiere, N. Bons and D. Albe-Fessard, "Spontaneous activity of rat dorsal horn cells in spinal segments of sciatic projection following transection of sciatic nerve or of corresponding dorsal roots", Neuroscience, vol. 94, pp. 217-228, 1999.

[26] N. Harrison and M. Simmonds, "Quantitative studies on some antagonists of N-methyl D-aspartate in slices of rat cerebral cortex", Br. J. Pharmacol., vol. 84, pp. 381, 1985.

[27] M. S. Lewicky, "A review of methods for spike sorting: the detection and classification of neural action potentials", Network: Computation of Neural Systems, vol. 9, pp. R53-R78, 1998.

[28] R. Chandra and L. M. Optican, "Detection, classification, and superposition resolution of action potentials in multiunit single-channel recordings by an on-line real-time neural network', IEEE Transactions on Biomedical Engineering, vol. 44, pp. 403-412, 1997. 